\title{
ARMENIEN UND IBERIEN ZWISCHEN ROM UND IRAN: WECHSELSEITIGE BEZÜGE, PARALlELE ENTWICKLUNGEN
}

\author{
Timo Stickler \\ iD http:/orcid.org/0000-0002-0335-9778 \\ Universität Jena
}

\begin{abstract}
Das Ziel der Aufsätze in diesem Sammelband ist es, die Kontexte aufzuzeigen, innerhalb derer sich die Geschichte (Groß-)Armeniens in den Jahrhunderten vor und nach Christus entfaltet hat. Es handelt sich um geographische, politische und kulturelle Kontexte.

Der folgende Beitrag unternimmt es hierbei, den Blick auf die Beziehungen zwischen Armenien und dem Königreich Iberien $\left(K^{\prime}\right.$ art $\left.{ }^{\prime} l i\right)$ zu richten. Iberien, gelegen zwischen Kolchis und Albanien, war die mittlere der drei antiken südkaukasischen Landschaften, die sich nördlich von Armenien zwischen dem Schwarzen und dem Kaspischen Meer erstreckten. Heute befinden sich hier die Staaten Georgien und Aserbaidschan.

In meinem Aufsatz richte ich den Fokus auf die wechselseitigen politischen Bezüge zwischen Armenien und Iberien in der Zeit der späten römischen Republik und der Kaiserzeit (1. Jh. v. Chr. bis 3. Jh. n. Chr.). Es geht mir insbesondere darum, auf Grundlage der griechischrömischen, der iranischen und der kaukasischen (armenischen, georgischen) Quellen strukturelle Gemeinsamkeiten herauszuarbeiten, die unsere Überlieferung insgesamt kennzeichnen.
\end{abstract}

Keywords: Armenia, Iberia.

\section{Bezüge zwischen der Geschichte Armeniens und Iberiens vom 1. Jh. v. bis zum 3. Jh. n. Chr.}

Seit dem Orientfeldzug des Cn. Pompeius Magnus 66-62 v. Chr. waren sämtliche Länder des südlichen Kaukasusraumes in die römische Interessenssphäre integriert. Die (griechisch-römische) Quellenlage ist dadurch günstiger als in der vorausgehenden Epoche. Doch auch jetzt fallen nur Schlaglichter unserer Überlieferung auf die Beziehungen zwischen Armenien und seinen nördlichen Anrainern, darunter Iberien. Die von diesen Schlaglichtern beleuchteten Ereigniskomplexe sind insbesondere die folgenden: ${ }^{1}$

1 Vgl. im folgenden die Überblicke über die kaukasisch-römischen Beziehungen seit Pompeius bei Dąbrowa 1989; Braund 1994, 152 ff.; Giardina 1996, 89 ff. und jüngst bei Preud'homme 2019, 457 ff.; vgl. auch Wheeler 2017. 
(1.) Der Kaukasusfeldzug des Pompeius 65 v. Chr. im Kontext des Dritten Mithradatischen Krieges: ${ }^{2}$ Nach der Unterwerfung des armenischen (Groß-)Königs Tigranes II. des Großen drang Pompeius bis nach Iberien vor und bezwang dessen König Artokes. Ein weiterer Vormarsch zum Kaspischen Meer wurde abgebrochen; Pompeius zog statt dessen über Kolchis an die Küste des Schwarzen Meeres zurück in Richtung des Königreiches Pontos.

(2.) Der Kaukasusfeldzug des P. Canidius Crassus 37/36 v. Chr. im Kontext der Vorbereitung des Partherkrieges des M. Antonius: ${ }^{3}$ Das Bündnis des Canidius Crassus mit dem armenischen König Artavasdes II. sowie seine Siege über den iberischen König Pharnabazos und den albanischen König Zober bereiteten die eigentliche Offensive gegen das Partherreich 36 v. Chr. vor, die dann allerdings scheitern sollte.

(3.) Die Erhebung Mithradates' I., des jüngeren Bruders des iberischen Königs Pharasmanes I., zum König von Armenien durch Kaiser Tiberius 35 n. Chr.: ${ }^{4}$ Der greise Prinzeps setzte in diesem Konflikt souverän und mit sparsamen Mitteln römische (und iberische) Interessen gegenüber dem parthischen Großkönig Artabanos II. durch. Letzterer hatte versucht, einen Arsakiden in Armenien zu installieren.

(4.) Die vorübergehende Installierung von Rhadamistos, einem weiteren Sohn von König Pharasmanes I. und Bruder Mithradates' I., als König von Armenien 51-54 n. Chr.. ${ }^{5}$ Eigentlicher Profiteur der hiermit einhergehenden Kabalen war der Vater, der zwar beide Söhne nacheinander opfern mußte, dafür aber mit Zustimmung der Römer südliche Grenzgebiete zu Armenien hin annektieren durfte, die im Verlaufe des 2. Jhs. v. Chr. an die Artaxiaden verlorengegangen waren. ${ }^{6}$ Armenien selbst hingegen fiel an den Arsakiden Tiridates I.

(5.) Die Teilnahme der Iberer an den Kämpfen Kaiser Trajans in Armenien und Mesopotamien im Gefolge des Partherkrieges 114-117 n. Chr.. ${ }^{7}$ Durch das aus Rom stammende Epitaph für den iberischen Prinzen Amazaspos ist die iberische Mitwirkung an den Kampfhandlungen prominent bezeugt. Es liegen weitere epigraphische Quellen vor, die intensive iberisch-römische Kontakte im 1. und 2. Jh. n. Chr. belegen. ${ }^{8}$

(6.) Konflikte zwischen dem iberischen König Pharasmanes II. und den Römern, die in einen ,Staatsbesuch“ in Rom im Jahre 141 n. Chr. mündeten: ${ }^{9}$ Der Alaneneinfall des Jahres 135 n. Chr. verdeutlicht die Bedeutung des Ibererkönigs sowohl für die römischen Kaiser Hadrian und Antoninus Pius als auch für den parthischen Großkönig Vologaeses III. Möglicherweise hat Pharasmanes II. durch die Ermöglichung der alanischen Invasion im Vorderen Orient seine Wichtigkeit als Bünd-

2 Hauptquellen: Plut. Pompeius 34, 1-36, 1; App. Mithr. 103 u. Cass. Dio 36, 54, 1-37, 5, 1. Die geographischen und topographischen Probleme des Kaukasusfeldzuges des Pompeius hat jüngst Bäbler 2019 untersucht.

3 Hauptquellen: Plut. Antonius 34, 10; Cass. Dio 49, 24, 1.

${ }^{4}$ Hauptquellen: Tac. ann. 6, 31-37; Ios. ant. Iud. 18, 96-105; Cass. Dio 58, $26,4$.

5 Hauptquelle: Tac. ann. 12, 44-51.

6 Vgl. Strab. 11, 14, 5: Möglicherweise handelt es sich um die Landschaften Gogarene und Chorzene.

7 Alleinige Quelle: $I G$ XIV $1374(=I G U R 1151=I G R R$ 1, $192=I K$ Estremo oriente $3=$ Anth. Pal. 3, 264).

8 Vgl. die Bauinschrift SEG 20, $112(=A E$ 1968, $145=I K$ Estremo oriente 1) und die sog. SerapeitisStele: $S E G$ 52, 1508 (= AE 1947, $125=S E G$ 16, $781=I K$ Estremo oriente 4).

9 Hauptquellen: HA Hadr. 13, 9; 17, 11 f. u. 21, 13; HA Pius 9, 6; Cass. Dio 69, 15, 2; 70, 15, 3; Arr. per. p. E. 11, 2 sowie das Fragment Og (Vidman 1982, 50) der Fasti Ostienses. Die komplexe Quellenlage ist jüngst von Hartmann 2019, 26 ff. nochmals eingehend gewürdigt worden. 
nispartner der Römer in Erinnerung rufen wollen. Spannungen im Verhältnis zu Hadrian stehen jedenfalls ostentative Freundschaftsbekundungen mit Antoninus Pius gegenüber.

(7.) Die Eroberung Armeniens und Südkaukasiens durch die Sāsāniden nach dem Sturz des Partherreiches 224 n. Chr.: ${ }^{10}$ Gegen starken einheimischen Widerstand erfolgte zwischen 252 und 262 n. Chr. die Inbesitznahme durch die persischen Großkönige, deren Herrschaft dann bis zum Frieden von Nisibis $298 \mathrm{n}$. Chr. andauern sollte.

Viele der angeführten Ereignisse werden in den Beiträgen dieses Tagungsbandes behandelt und müssen deshalb nicht noch einmal im einzelnen analysiert werden. ${ }^{11}$ Ich selbst werde im folgenden nur auf den letztgenannten Stichpunkt, die Eroberung Armeniens und Südkaukasiens durch die Sāsāniden nach dem Sturz des Partherreiches zwischen 252 und $262 \mathrm{n}$. Chr., ausführlicher eingehen. Er soll als exemplum für die Quellenproblematik dienen, die die antike Geschichte des südkaukasischen Raumes insgesamt aufwirft.

Blickt man auf die Quellen für die Bezüge zwischen der armenischen und der iberischen Geschichte zwischen dem 1. Jh. v. und dem 3. Jh. n. Chr., so lassen sich zunächst zwei allgemeine Aussagen treffen. Erstens ist unsere literarische und nichtliterarische Überlieferung in der Regel mehr als dürftig und zweitens nimmt sie nie von den Verhältnissen in Armenien oder Iberien selbst Ausgang, sondern von anderen, übergeordneten Themen, die im Fokus der Autoren lagen.

In der Regel handelt es sich nämlich um beiläufige Notizen, die das eigentliche Geschehen, kriegerische Auseinandersetzungen zwischen den Römern und ihren parthischen und persischen Gegnern, illustrieren und mit zusätzlichen Einzelheiten bereichern. Dies ist im überwiegenden Teil unseres griechisch-römischen Quellenmaterials der Fall, zumal in ereignisgeschichtlich fokussierten oder gattungsbedingt gerafften Darstellungen. ${ }^{12}$ Oder es handelt sich um Passagen, die der Verherrlichung oder Herabsetzung eines Protagonisten dienen und aus diesem Grunde den antiken Autor veranlaßten, länger als üblich bei armenischen oder iberischen Gegenständen zu verweilen. Dies ist z. B. in den einschlägigen Passagen der plutarchischen Pompeius-Vita der Fall. Sie basieren auf der Darstellung des zeitgenössischen Historikers Theophanes von Mytilene, eines engen Gefolgsmannes des späteren Triumvirn. Ganz offensichtlich soll hier das Bild des Pompeius als eines neuen Alexander Magnus gezeichnet werden, der es dem großen Vorbild durch seinen Feldzug in den Peripherien der Ökumene in mehrfacher Hinsicht gleichgetan habe - hinsichtlich des militärischen Erfolgs, hinsichtlich der geographischen Reichweite, sogar im Hinblick auf den Kontakt mit der Welt des Mythos. ${ }^{13}$ Tacitus widmet dem Einblick in die Konflikte und Wirrnisse der kaukasischen Politik deshalb so breiten Raum, weil er auf diese Weise dem Auftritt seines ,Helden ' Cn. Domitius Corbulo auf dem orientalischen Kriegsschauplatz in adäquater Weise den Boden bereiten kann. ${ }^{14}$

$10 \mathrm{Zu}$ den Hauptquellen für die Ereignisse siehe ausführlich unten, S. 193ff.

11 Vgl. hierzu die Beiträge von Dąbrowa, Daryaee und Cereti.

12 So z. B. bei Tacitus, Cassius Dio, den spätantiken Breviarien, Johannes Zonaras etc.

13 Vgl. bereits App. Mithr. 478 sowie Plut. Pompeius 34, 7; 38, 4; dazu Heftner 1995, 53 ff.

14 In diesem Sinne Heil 1997, 40 ff. Kühnert 1980, 39 betont die Kaiserkritik, die den betreffenden Passagen in Tacitus' Annales zugrunde liegt: „Der ganze Bericht über die iberisch-armenischen Kämpfe erhält durch diesen Rombezug eine höchst aktuelle Bedeutung: das unwürdige Verhalten der Vertreter der 
Umgekehrt ist es wiederholt im Sinne des Autors der Historia Augusta, Kaiser Hadrian in ein schlechtes Licht zu setzen; so erklärt sich der negative Duktus der Passagen, die das Verhältnis zwischen diesem und dem iberischen Klientelkönig Pharasmanes II. zum Thema haben. ${ }^{15}$

Wo die beiden genannten Aspekte - Beiläufigkeit oder eine Fokussierung auf bestimmte Personen -, aus welchen Gründen auch immer fehlen, tappen wir im dunkeln, so etwa bei den Ereignissen im 3. Jh. n. Chr. Weder die Eroberung des südlichen Kaukasusraumes durch die Sāsāniden noch dessen Rückeroberung durch die Tetrarchen ist gut belegt. In diesen Fällen stellen iranische Quellen wie die dreisprachige Inschrift Šābuhrs I. an der Ka 'ba-i Zardušt, die Inschriften des zoroastrischen Priesters Kirdīr oder die Inschrift Narsehs am Monument von Pāikūlī ein ungenügendes, aber dennoch willkommenes Substitut dar. Wir werden darauf sogleich zurückkommen.

Auf römischer und iberischer Seite zufällig erhalten gebliebene Inschriften zeigen, welche Dimensionen des Wissens uns entgehen, aber weil sie so wenig zahlreich sind, werfen sie oft ebenso viele Fragen auf, wie sie beantworten. Man vergleiche in diesem Zusammenhang nur die andauernden Forschungskontroversen um die Datierung und Auswertung der sogenannten Serapeitis-Stele. ${ }^{16}$ Das gleiche gilt im übrigen für archäologisch überlieferte Artefakte aus iberischen Gräbern, die Kontakte zwischen den dortigen Eliten und Rom bezeugen. Auch sie sind mehr oder weniger Unikate, die sich einer sicheren Interpretation entziehen. ${ }^{17}$

Die griechisch-römische Überlieferung zur Geschichte des südlichen Kaukasusraums zwischen dem 1. Jh. v. und dem 3. Jh. n. Chr. - sei sie literarisch, epigraphisch oder archäologisch dokumentiert - ist also defizitär und läßt uns den ereignisgeschichtlichen Verlauf nur in groben Zügen erkennen. Hilft demgegenüber die einheimische kaukasische Überlieferung? ${ }^{18}$ Hierzu nun unser Fallbeispiel, die Eroberung des südlichen Kaukasusraumes durch die Sāsāniden um 252/62 n. Chr.

römischen Macht und darüber hinaus die unfähige römische Außenpolitik des Claudius bzw. des Nero werden implicite scharf kritisiert und verurteilt."

15 Siehe Hartmann 2019, 39 f. Hartmann hält eine tatsächliche Konfrontation zwischen Kaiser Hadrian und König Pharasmanes II. für eher unwahrscheinlich; sie habe dem Historia-Augusta-Autor lediglich dazu gedient, ,den hochmütigen Orientalen dem schwachen Kaiser“ (ebd., 39) gegenüberzustellen.

16 Siehe die Bibliographie unter $I K$ Estremo oriente 4. Ältere Forschungsüberblicke bei Bosworth 1977, 230 ff. und Braund 1994, 212 ff.; zuletzt Hartmann 2019, 47 Anm. 64 und Preud'homme 2019, 604 ff.

17 Vgl. z. B. den Silberteller aus einem Grab in Armazisq'evi nahe Mc'xet'a mit der Nennung eines

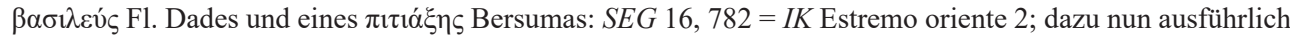
Coert - Schmitt 2019; vgl. auch - mit anderen Schlußfolgerungen - Preud'homme 2019, 143 f. u. 619 ff.

18 Dabei kann es nicht nur um den Nutzen der kaukasischen Überlieferung für die Klärung der Ereignisgeschichte gehen. Ganz im Sinne von Traina 2018, 109 gilt: „In any case, the scope of this paper is not to consider the historical value of Armenian texts, but to examine their approach to the rise of the Sasanians, a period that had important repercussions on the geopolitical balance of the region." 


\section{Die Sicht der kaukasischen (armenischen, georgischen) Quellen}

Der Vorgang der Integration Armeniens und Iberiens in den sāsānidischen Machtbereich nach der Mitte des 3. Jhs. n. Chr. ist alles andere als klar, sowohl hinsichtlich der Datierung als auch des Verlaufs und der Folgen. ${ }^{19}$

Für das 3. Jh. n. Chr. beobachten wir in den griechisch-römischen Quellen naturgemäß eine Konzentration auf die persisch-römische Konfrontation, mit dem dramatischen Höhepunkt der Niederlage und Gefangennahme Kaiser Valerians in der Kampagne von 260 n. Chr. Laut Historia Augusta waren die Armenier, Iberer und Albaner selbst nach dem Eintritt der Katastrophe noch bereit, die Römer und danach die mit diesen verbündeten Palmyrener zu unterstützen. ${ }^{20}$ Grundsätzlich werden die kaukasischen Verhältnisse nur am Rande einbezogen. Bei Cassius Dio etwa wird eine Niederlage Ardašīrs I. gegen die armenischen Arsakiden und ihre Verbündeten erwähnt, ${ }^{21}$ bei Zonaras (bzw. der ihm zugrundeliegenden Leoquelle) findet sich ein Hinweis auf die Eroberung Armeniens durch Šābuhr I.;22 bei beiden Autoren allerdings findet sich gerade keine Erwähnung der Iberer.

Die iranischen Quellen bieten folgendes Szenario: In den Res gestae divi Saporis um 260/62 n. Chr. wird der armenische König Hormizd-Ardašīr erwähnt. ${ }^{23}$ Er war der leibliche Sohn Šābuhrs I. und als Hormizd I. um 270/72 n. Chr. dessen Nachfolger als Großkönig des Sāsānidenreiches; demgegenüber ist der von der Historia Augusta genannte rex Armeniorum Artabasdes wohl als fiktiv zu betrachten. ${ }^{24}$ Ebenfalls in den Res gestae wird ein iberischer König (H)amāzāsp erwähnt, der den Sāsāniden untertan und steuerpflichtig war. ${ }^{25}$ Sowohl hier als auch in den Inschriften Kirdīrs und in der Inschrift von Pāikūlī 293/94 n. Chr. firmiert Iberien als Land (̌̌ahr, ع̌ $\theta v o \varsigma)$ des Großkönigs, seine Könige sind šahrdārān, ranghohe Dynasten des Sāsānidenreiches. ${ }^{26}$ Dieser Zustand scheint jedoch noch nicht für die Zeit des Vorgängers Šābuhrs I., diejenige des Reichsgründers Ardašīr I., zu gelten. Erst unter Šābuhr, vermutlich 252 n. Chr., erfolgt die Eroberung Armeniens durch die Sāsāniden, danach, auf jeden Fall vor 262 n. Chr. dann auch diejenige Iberiens. ${ }^{27}$

19 Einen aktuellen Überblick zu den Vorstößen Šābuhrs I. auf das Reichsgebiet in den 250er und 260er Jahren n. Chr. geben Huttner 2008, 214 f. u. 218 ff. sowie Goltz, Hartmann 2008, 234 ff. u. 248 ff.; siehe auch Hartmann 2019, 40 ff. Die kaukasische Perspektive nimmt Kettenhofen 2008, 480 ff. ein; vgl. auch Christensen 1944, 218 ff.; Chaumont 1976, 158 ff.; Braund 1994, 238 ff.; Garsoïan 1997, 71 ff.; Winter, Dignas 2001, 41 ff. u. 93 ff.; Mosig-Walburg 2009, 43 ff. u. 79 ff.; Rapp 2014, 240 ff. sowie nun Preud'homme 2019, $468 \mathrm{ff}$.

20 HA Val. 3 f. Einordnung dieser Passage bei Hartmann 2019, 40 ff.

21 Cass. Dio 80, 3, 3

22 Zon. 12, 21 (p. 589 f. Pinder - Büttner-Wobst).

23 ŠKZ § 33.5 u. 37.1; vgl. auch HA tyr. trig. 2, 2.

24 HA Valer. 3. In diesem Sinne auch Kettenhofen 2008, 483 f. und Hartmann 2019, 44.

25 ŠKZ § 44.4; vgl. auch ŠKZ § 2.11.

26 Vgl. ŠKZ § 2.11; KSM 18 f.; KNRm 39; KKZ 12 u. NPi § 92.

27 So die überzeugende Rekonstruktion von Kettenhofen 2008, 481 f.; 490 f.; vgl. auch Hartmann 2019, $45 \mathrm{ff}$. 
Blicken wir nun auf die armenischen Quellen; sie zeichnen das folgende Bild:28 Agat $^{{ }}$angełos ${ }^{29}$ enthält den Bericht über einen Sieg der Armenier unter ihrem König Xosrov über Großkönig Artašīr (gemeint ist Ardašīr I.). Ausgangspunkt der Geschichte ist der Sturz der parthischen Arsakiden durch die persischen Sāsāniden. Die Armenier werden dabei von den Albanern (Aluank'), Iberern (Virk') und Hunnen (Honk') unterstützt und kontrollieren über die Pässe von Darial und Derbent die Kontakte ins Nordkaukasusgebiet. In der Folgezeit feiert Xosrov über rund zehn Jahre hinweg große Erfolge gegen die Sāsāniden. Er unternimmt Plünderungszüge bis nach Mesopotamien (Asorestan) und bis vor die Residenzstadt Ktesiphon. Dabei wird er durch Völker aus dem Bereich des Kaukasus und des Kaspischen Meeres (Atuank' / 'A $\lambda \beta \alpha v o i ́$,

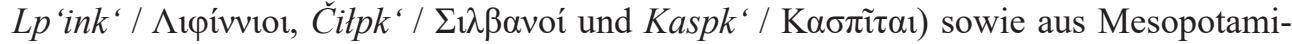

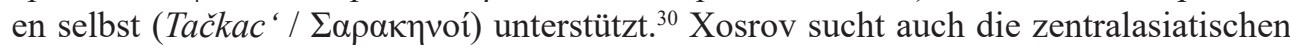
Kušān (K’ušakan) als Bundesgenossen zu gewinnen. Sein Krieg mit den Sāsāniden weitet sich nachgerade zu einem antiken, Weltkrieg' aus. Dennoch behält die Auseinandersetzung immer den Charakter einer Vendetta unter vornehmen iranischen Adelsfamilien. Xosrov agiert als Arsakide, von einem verräterischen Arsakiden, Anak aus dem Hause Surēn Pahlav, wird er heimtückisch ermordet. Damit ist die Peripetie erreicht: Erst nach der Ermordung Xosrovs und der Flucht von dessen Sohn Trdat (dem künftigen König Tiridates III., der einst zum Christentum übertreten wird) ins römische Reich erfolgt die Eroberung Armeniens durch die Sāsāniden.

Auch bei Movsēs Xorenac ${ }^{i} i^{31}$ wird ein armenischer König Xosrov genannt, der in Opposition zu den Sāsāniden gestanden habe. Bei ihm ist das Interesse des Autors an der Positionierung der iranischen Adelsgeschlechter infolge des Sturzes der Arsakiden sogar noch deutlicher erkennbar als bei Agat ${ }^{\circ}$ angełos. Xosrov kämpft in der Folge, unterstützt vom römischen Kaiser Philippus Arabs, erfolgreich gegen Großkönig Artašīr. Dennoch bleiben die meisten iranischen Familien den Sāsāniden gegenüber treu. Bei Movsēs wird die Krise Roms in der Soldatenkaiserzeit stärker in die Geschehnisse miteinbezogen. Dennoch bleibt die primär orientalische Perspektive gewahrt; der armenische König Xosrov treibt die Sāsāniden unter Artašīr bis nach Indien zurück. Am Ende wird, wie bei Agat ${ }^{\circ}$ angełos, Xosrov durch Anak aus dem Hause Surēn Pahlav nach 48 Regierungsjahren ermordet.

Werfen wir nun einen Blick auf die georgischen Quellen; hier stellt sich der Ereigniszusammenhang folgendermaßen dar: Die einschlägigen Passagen in $K^{\prime}$ art'lis $C^{\prime}$ xovreba ${ }^{32}$ entstammen einer alten Schicht der Chronik; sie führen auf jeden Fall in die Zeit vor 813 n. Chr. zurück. ${ }^{33}$ Angeblich greifen sie auf eine persische Schriftquelle, das „Leben der Perser“ ( $C^{\prime}$ 'xorebay sparst ' $a$ ) zurück, das mit dem verlorenen mittelpersischen „Herrenbuch“ (Xvadāy-nāmag) identisch sein könnte. ${ }^{34}$ Auch in $K^{\prime}$ art 'lis

28 Vgl. hierzu auch Traina 2007 und dens. 2018.

29 Agat ${ }^{\circ}$ angełos 18-36 (p. 34-53, Thomson 1976).

30 Zur Identifizierung der betreffenden Völker siehe Thomson 1976, 455 f. sowie Preud'homme 2019, 22 u. 225.

31 Movs. Xoren. 2, 71-76 (p. 218-224, Thomson 1978).

32 K'art'lis C'xovreba 59-64 (p. 69-75, Thomson 1996a).

33 Zur Datierung der unterschiedlichen Textschichten von $K^{\prime}$ art 'lis C'xovreba siehe Rapp 2014, $172 \mathrm{ff}$.

34 Vgl. K'art'lis C'xovreba 59 (p. 70, Thomson 1996a); dazu ausführlich Rapp 2014, 191 ff. 
$C^{\prime}$ xovreba werden vorübergehende Erfolge der verbündeten Armenier und Iberer gegen die Sāsāniden beschrieben. Insgesamt ist eine große Nähe zu Agat'angełos erkennbar. Mit Asp`agur erlischt die einheimische iberische Königsdynastie der Pharnabaziden, sein Nachfolger Mirian wird von der georgischen Tradition als erster Chosroïde geführt. Angeblich war er ein Sohn des persischen Großkönigs K'asre Anušarvan. Der Übergang Iberiens an die Sāsāniden erfolgt schließlich ohne Kampf, vielmehr durch einen Friedensschluß mit Heiratsbündnis. Die iberischen Großen handeln beim Abschluß dieses Bündnisses ausdrücklich unter dem Eindruck der Eroberung Armeniens durch die Perser. Auch in der georgischen Überlieferung herrscht zum Teil Konfusion bei den Namen der persischen (K'asre Anušarvan bzw. Ardabir) und der einheimischen Herrscher (der Armenier Kosaro, die Iberer Asp'agur und Mirian). Des weiteren gibt es Anachronismen bei den Namen der mitkämpfenden Völker, nämlich der Osseten (Ovsni), Chasaren (Xasarni) und Leken (Lekni). Die ersten beiden verweisen auf den reiternomadisch geprägten Raum nördlich des Kaukasus, die zuletzt Genannten auf das heutige Dagestan. ${ }^{35}$

\section{Die kaukasische Überlieferung in ihrem Verhältnis zur übrigen: Auswertung}

Auffallend sind im Hinblick auf die Auswertung unseres Befundes zunächst die Gemeinsamkeiten der armenischen und georgischen Überlieferung. Die Unterwerfung sowohl Armeniens als auch Iberiens unter die Herrschaft der Sāsāniden wird nicht geleugnet. Sie vollzieht sich durch Kampf (Armenien) bzw. durch einen Vertragsschluß (Iberien) und ist mit dem Ende der bisherigen Dynastie (Arsakiden, Pharnabaziden) verbunden. Die Heereszüge der Sāsāniden in den südlichen Kaukasus werden mit dem Sturz der Arsakiden in Zusammenhang gebracht. ${ }^{36}$ Die beschriebenen Kämpfe nehmen nachgerade den Charakter einer adeligen Vendetta an. Dieser Aspekt tritt gegenüber demjenigen eines irgendwie gearteten ,nationalen' Freiheitskampfes der Armenier bzw. Iberer / Georgier eindeutig in den Vordergrund. Der Kampf gegen die Sāsāniden wird auch in ,welthistorische“ Zusammenhänge gestellt, sei es in westliche Richtung (Rom), östliche (Zentralasien: Kušān; Indien) oder nördliche (Nordkaukasusgebiet: ,Hunnen“ etc.).

Sachliche Fehler gibt es, gerade hinsichtlich des Personals. Besonders signifikant ist das im Hinblick auf den wohl fiktiven König Chosroës II. (Xosrov / Kosaro), der jahrzehntelang über Armenien regiert, jahrelang erfolgreich gegen die Sāsāniden gekämpft und schließlich nur durch einen heimtückischen Mord habe beseitigt werden können. ${ }^{37}$

$35 \mathrm{Zu}$ den Anachronismen hinsichtlich der Völkernamen in K'art'lis C'xovreba siehe Rapp 2014, 130 Anm. 129, mit Bezug auf die Chasaren ebd., 199 ff.; vgl. hierzu auch Preud'homme 2019, 223 Anm. 1242. Die Lokalisierung der Leken in Dagestan geht bereits aus K'art'lis C'xovreba 5 f. (p. 6, Thomson 1996a) hervor; hierzu auch Heftner 1995, 254 unter Verweis auf Markwart 1931, 95 f.

36 Diesen Aspekt hebt Traina 2018, 114 f. hervor und sieht darin ,an exemplary case of “invention of tradition"“; vgl. auch dens. 2015, $160 \mathrm{f}$.

37 Zum armenischen König Chosroës II. (Xosrov / Kosaro) siehe die überzeugenden, wenn auch desillusionierenden Ausführungen von Kettenhofen 2008, 479 ff.; Chaumont 1976, 159 ff.; Garsoïan 1997, 71 ff. und Preud'homme 2019, 469 ff. wissen um die Problematik der armenischen Überlieferung, versuchen sie aber dennoch auf die eine oder andere Weise in ihre Darstellung der Ereignisgeschichte zu integrieren. 
Doch solche ,Fehler' sind keinesfalls nur auf die armenische und georgische Überlieferung beschränkt. Man vergleiche nur das Auftreten des rex Armeniorum Artabasdes in der Historia Augusta; auch er ist lediglich um seiner Funktion im Kontext der Vita Valerianorum duorum willen erfunden worden, vermochte er doch auf diese Weise die Loyalität der kaukasischen Könige zum römischen Reich auch nach der Gefangennahme Kaiser Valerians 260 n. Chr. zu ,bezeugen ${ }^{38}$ Nicht anders haben es die armenischen und georgischen Autoren der Spätantike und des frühen Mittelalters gemacht; das historische Bild, das sie zeichnen, ist nur reicher an Einzelheiten und (vermeintlichen) Details.

Das zeitgenössische historische Umfeld der armenischen und georgischen Autoren der Spätantike und des frühen Mittelalters wirkt immer wieder auf ihre Darstellung zurück, wodurch - mit zunehmender zeitlicher Distanz - die Zahl der sachlichen Fehler ansteigt, ja ansteigen muß, zumal das Geschehen mit immer mehr Details angereichert wird. ${ }^{39}$ Gut erkennbar ist das an der Verwendung des Xusrō / K'asre-Namens für den sāsānidischen Großkönig in der georgischen Überlieferung, ${ }^{40}$ des weiteren an den auffallenden Anachronismen bei den Völkernamen. ${ }^{41}$

Der Prozeß der Ablösung von der Faktentreue in unserem modernen, aber auch im klassisch-antiken, thukydideischen Sinne ${ }^{42}$ ist schon bei Agat ${ }^{e}$ angełos in vollem Gange. Der fiktive Xosrov ist auch bei ihm schon armenischer König; ,Hunnen“ unterstützen die Armenier im Kampf. Aber der Prozeß verstärkt sich immer mehr in der Nachantike: Xosrov regiert schließlich genau 48 Jahre, der sāsānidische Großkönig flieht vor ihm bis Indien, Mirian III. ist ein Sohn des persischen Großkönigs etc. Nur die zeitgenössische dokumentarische iranische Überlieferung kann im einzelnen Aufschluß über den tatsächlichen Verlauf der Ereignisgeschichte bieten, aber ausgerechnet sie hat keinen Eingang in die armenische und georgische Historiographie gefunden. Statt dessen haben sich, so könnte man konstatieren, ,alternative Fakten' durchgesetzt. Warum ist das so? ${ }^{43}$

\section{Bezüge zwischen der kaukasischen und der iranischen historischen Tradition}

Strukturell basiert die die kaukasische (armenische und georgische) Geschichtsschreibung nicht allein auf griechischen Vorbildern, ${ }^{44}$ sondern ist auch historischen Traditionen

38 Siehe HA Val. 3.

39 Zum Prinzip des fama crescit eundo insbesondere im hagiographischen Kontext siehe Heid 1991, bes. $105 \mathrm{f}$.

40 Hierzu Rapp 2014, 41 f., der im Xusrō / $K$ 'asre-Namen der georgischen Überlieferung ,a generic designation for all šăhan šāhs" sieht (ebd., 42), analog zu Caesar / Kaiser.

41 Siehe abermals Rapp 2014, 130 Anm. 129.

42 Vgl. das sog. Methodenkapitel Thuk. 1, 22.

43 Diese Frage ist zentral für das Verständnis der armenischen resp. kaukasischen Geschichtsschreibung; in diesem Sinne auch Traina 2002, 456: „Ne risulta un prodotto storiografico alquanto scoraggiante ma, al tempo stesso, affascinante per la sfida presentata da questa storiografia ,meticcia‘, ben più sofisticata di quanto non si voglia ammettere, a decifrarne la complessità.“

44 Dieser Einfluß ist selbstverständlich vorhanden und wird z. B., mit Blick auf Movsēs Xorenac ${ }^{\circledR} i$, von Terian 2001-2002, bes. 125 ff. stark betont. Allgemeine Kennzeichen der armenischen Geschichtsschreibung auch bei Traina 2015, der armenischen und georgischen Geschichtsschreibung bei Thomson 1996c. 
verwandt, wie sie mutmaßlich in das mittelpersische „Herrenbuch“ (Xvadāy-nämag) eingegangen sind. ${ }^{45}$ Unser Problem besteht allerdings darin, daß wir den genauen Zuschnitt dieser Traditionen nicht mehr unmittelbar nachvollziehen können, denn das mutmaßlich in der ausgehenden Sāsānidenzeit zusammengestellte „Herrenbuch“ ist nicht direkt auf uns gekommen. ${ }^{46}$ Seine Inhalte sind sehr wohl in die arabische Historiographie, etwa in die „Annalen“ at-Ṭabarīs, eingegangen, desgleichen in Firdausīs neupersisches Epos „Buch der Könige“ ( $\check{S} \bar{a} h n \bar{a} m e) .{ }^{47}$ Aber worin diese genau bestanden, ist in der Forschung umstritten. Alireza Shahpur Shahbazi hat aus den dürftigen Hinweisen unserer Überlieferung ein vergleichsweise anspruchsvolles Profil des „Herrenbuchs“ rekonstruiert und glaubt sogar dreier Versionen dieses Werkes („royal“, „priestly“, „heroic“) nachweisen zu können. ${ }^{48}$ Demgegenüber sind die Schlußfolgerungen von Jaakko Hämeen-Anttila viel zurückhaltender. Er denkt an ein eher kleines Buch, das eine Königsliste und wenige, in erster Linie für die Erstellung einer Chronologie wichtige Einzelheiten enthalten habe. $^{49}$

Die scharfen Gegensätze bei der Bewertung des „Herrenbuches“ und seiner Inhalte ergeben sich daraus, daß wir auch über die historischen Traditionen Irans, aus denen dieses geschöpft haben könnte, nicht viel aussagen können. Die Forschung operiert mit den immer gleichen tatsächlichen oder vermeintlichen ,Quellen', aus denen sich die historische Tradition Irans gespeist haben mag: Archivalien, epische Erzählungen, bauliche Reste aus der Vergangenheit, mündliche Tradition (fußend auf einer origo und adeligen Genealogien), potentiell auch die biblisch-jüdische Überlieferung. ${ }^{50}$ Aber ob und wie mittelpersische Autoren aus diesen Ingredienzien so etwas wie eine „Sasani-

45 Auf dieser Hypothese fußt die Monographie von Rapp 2014, zusammenfassend 353 ff.; vgl. schon dens. 2003. Die Beeinflussung der kaukasischen Geschichtsschreibung durch ihre iranische Umwelt ist schon lange erkannt und an Beispielen dargestellt wurden; dazu Garsoïan 1996. Vgl. in diesem Zusammenhang beispielhaft Garsoïan 1982 zur Symbolik des Ebers bzw. Schweines in der Bekehrungsgeschichte Tiridates' III. bei Agat' angełos. Auch Thomson 1982, der die Vielfalt der Einflüsse auf die seit dem 5. Jh. n. Chr. entstehende armenische Literatur herausarbeitet, charakterisiert in seinem Resümee ebd., 148 die betreffenden Autoren als ,,very conscious of belonging to a society that was foreign to the city culture of the Hellenistic world and of sharing ancient traditions that derived from the Iranian world."Wie Thomson 1996b, 41 sieht auch Rapp 2014, 361 die georgische Überlieferung stärker im der iranischen Tradition verankert als die armenische: „Important traces of the Xwadāy-nāmag tradition are evident in some of these Armenian sources, but the Iranian epic did not serve as their main model." Für Georgien hingegen postuliert er, analog zum Xwadāynāmag, ein Hambavi mep'et' $a$, das die Grundlage eines Teils der uns heute vorliegenden Überlieferung gebildet habe.

46 Allgemeine Charakteristika der iranischen historischen Überlieferung bei Shahbazi 2004, zusammenfassend 328: „Iranian historiography was moralistic, providential, apocalyptic, rather particularistic, and utilitarian.“ Diesen letzten Gesichtspunkt betont auch Yarshater 1983, 369: „Thus the (scil. Iranian) historiographer, far from being an impartial investigator of facts, was an upholder and promoter of the social, political, and moral values cherished by the Sasanian élite."Vgl. auch Christensen 1944, $50 \mathrm{ff}$.

47 Siehe ausführlich Hämeen-Anttila 2018; ferner Pourshariati 2008; 34 f.; 171 ff. und Rapp 2014, bes. $353 \mathrm{ff}$. Vgl. in diesem Zusammenhang insbesondere die konzise Einführung in die Problematik von Hoyland 2018, 1 ff., bes. $18 \mathrm{ff}$.

48 Siehe Shahbazi 1990, bes. $215 \mathrm{ff}$.

49 So Hämeen-Anttila 2018, zusammenfassend ebd., $213 \mathrm{ff}$.

50 Siehe in diesem Zusammenhang die akribische Zusammenstellung von Shahbazi 1990, 209 ff. und Daryaee 2001-2002, 4 ff. Einen Überblick über die mittelpersische Literatur gibt Cereti 2001. 
an historiography ${ }^{651}$ fabriziert haben, bleibt, recht besehen, unklar. Robert G. Hoyland hat vor kurzem gute Argumente dafür zusammengetragen, daß in der ausgehenden Sāsānidenzeit neben dem „Herrenbuch“, das auch er in erster Linie als knappe, chronologisch angeordnete offizielle Königsliste auffaßt, weitere Texte existiert hätten, die die Taten von Königen und anderen hochgestellten Persönlichkeiten in ausführlicherer Weise, als ,epic tales and romances“ ${ }^{52}$ thematisiert hätten. Beide Formen des ,historical writing about pre-Islamic Persia" ${ }^{653}$ seien bald miteinander verbunden und in einer Vielzahl von mittelpersischen und arabischen Texten weitertradiert worden, die dann wiederum auf unterschiedliche Weise von der frühislamischen Geschichtsschreibung rezipiert worden seien. Hoyland selbst bezeichnet seine Schlußfolgerungen als ,very tentative“ 54 aber was sie in jedem Falle deutlich machen, ist doch, daß man sich in der Frage der historischen Tradition Irans von der Fixierung auf das mittelpersische „Herrenbuch“ freimachen darf und freimachen muß, wenn es darum geht, den Umgang der Perser mit ihrer Vergangenheit in der späten Sāsānidenzeit besser zu verstehen. Womit wir da konfrontiert werden, ist in vielerlei Hinsicht anders als das, was wir von der griechischrömischen Geschichtsschreibung gewohnt sind; es erinnert eher an mythische Heroengeschichten und epische Erzählungen. Aber gerade das ist der Umstand, der es uns meines Erachtens erlaubt, die iranische historische Tradition mit der kaukasischen zu verbinden. Denn alle Elemente, die letztere auszeichnen - die Entwicklung der Darstellung entlang einer Königsliste, aber gegebenenfalls eben auch eine resolute Loslösung von der Ereignisgeschichte; man denke nur an die Heldentaten des Armenierkönigs Chosroës II. (Xosrov / Kosaro) - finden sich darin wieder.

Touraj Daryaee hat jüngst Gesichtspunkte zusammengestellt, die in ihrer Summe seiner Ansicht nach darauf hinweisen, daß sich die Sāsāniden im Verlaufe der Spätantike - er denkt insbesondere an die Zeit seit Šābuhr II. (309-379 n. Chr.) - zusehends auf ihre , avestische' (und damit zoroastrische) Abkunft besonnen hätten. Aus diesem Grunde auch hätten sie an die mythischen Pīšdādiden und Kayāniden, nicht so sehr an die historischen Achaimeniden angeknüpft. ${ }^{55}$ Der Befund ist aufschlußreich: Wenn Daryaees Deutung zutrifft, könnten wir als Ausgangspunkt des sāsānidischen historischen Schrifttums die (Rück-)Besinnung der persischen Großkönige auf eine religiös fundierte, mythische Vergangenheit bestimmen, nicht so sehr auf eine ,historische“. Daryaee spricht selbst zutreffend von einer „sacred historiography“, die eine ,,actual history“ griechischrömischen Zuschnitts weitgehend habe ausblenden können. ${ }^{56}$ Mit Recht stellt er im Falle der Bewertung der iranischen Überlieferung die Begriffe „memory“ und „history“ einander gegenüber und vermeidet eine Opposition zwischen (vermeintlicher) ,amnesia“ (etwa gegenüber den Achaimeniden) und ,history“. ${ }^{57}$ All dies ließe sich auch auf die

51 Hämeen-Anttila 2018, 229.

52 Hoyland 2018, 22; vgl. auch ebd., 21, wo er „,chronological compilations of essential information about kings“ den ,epic narratives about specific persons, especially kings, and their heroic deeds“ gegenüberstellt.

53 Ebd., 23.

54 Ebd.

55 Siehe Daryaee 2001-2002, bes. $10 \mathrm{ff}$.

56 Ebd., $11 \mathrm{f}$.

57 Ebd., 11. Dazu passen gut die Beobachtungen von Huyse 2008, 152, der den besonderen Charakter der iranischen Überlieferung darauf zurückführt, daß sie lange Zeit nur mündlich tradiert worden sei: „,The curiosity of the past for curiosity alone, without a direct link to the present, is considered to be vain in oral 
armenische und georgische Geschichtsschreibung übertragen, sogar in mehrfacher Hinsicht. Denn sowohl die Herleitung von eponymen Heroen wie Hayk und K'art'los als auch deren (nachträgliche) Verknüpfung mit der biblischen Geschichte lassen sich gut mit dem beschriebenen sāsānidischen Schema in Übereinstimmung bringen. ${ }^{58}$ Die Christianisierung Armeniens und Iberiens im Verlaufe des 4. Jhs. n. Chr. erfolgte zwar vom römischen Reich und nicht von Iran aus, sie verstärkte gleichwohl den beschriebenen Impuls noch. Als die kaukasischen Autoren, ausgerüstet mit eigens im Zuge der Christianisierung geschaffenen epichorischen Alphabeten, nun darangingen, die Vergangenheit ihrer Heimatländer aufzuzeichnen, räumten auch sie der religiös fundierten Vergangenheit den Primat ein - mit allen Folgen für eine wie auch immer geartete historische Korrektheit im thukydideischen Sinne. ${ }^{59}$

\section{Die Theorie einer handlungszentrierten Geographie von Benno Werlen}

Aus dem bisher Gesagten geht hervor, daß die kaukasische (armenische, georgische) Überlieferung also gerade nicht dazu dienen kann, die Ereignisgeschichte, in unserem Falle das Vordringen der Sāsāniden nach Armenien und Iberien im 3. Jh. n. Chr., korrekt zu rekonstruieren. Versuche, sie dennoch diesbezüglich miteinzubeziehen, zwingen entweder dazu, miteinander Unvereinbares nebeneinander stehenzulassen oder in einer Art und Weise zu harmonisieren, die letztlich nicht überzeugen kann. Ernst Kettenhofens Urteil über das Geschichtswerk des Movsēs Xorenac 'i, es sei „für eine historische Rekonstruktion weithin unbrauchbar" ${ }^{60}$ ist vor diesem Hintergrund schmerzlich, aber nur ehrlich. Dennoch bleibt die Frage bestehen, wie man aus Überlieferungen dieser Art einen Nutzen ziehen kann? Ich will im folgenden in einem letzten Schritt versuchen, das Konzept einer handlungszentrierten Geographie (action-oriented social geography), wie es Benno Werlen entwickelt hat, für unsere Fragestellung fruchtbar zu machen. ${ }^{61}$

Werlen sieht ,Raum‘ und ,Gesellschaft' eng aufeinander bezogen. Dies sichtbar zu machen, ist das Ziel seiner Konzeption von Sozialgeographie. Es geht ihm dabei gera-

societies. This is what explains the 'structural amnesia' Ehsan Yarshater spoke about a quarter of a century ago." Huyse bezieht sich hier vermutlich auf Passagen wie Yarshater 1983, 389 (,historical amnesia“).

58 Vgl. in diesem Zusammenhang Rapp 2014, 187 ff., der in dieselbe Richtung denkt.

59 Dies um so mehr, als die politisch-militärischen Erfahrungen im 5. Jh., kulminierend in der armenischen Niederlage von Avarayr 451 n. Chr. gegen die nichtchristlichen Sāsāniden, traumatisch waren. Entsprechend urteilt Garsoïan 1994, 125 über die armenische Geschichtsschreibung in ihrer formativen Phase: „From earliest times, therefore, its ideological position was to pull it of necessity in the opposite direction from political realism.“

60 Kettenhofen 1995, 165; vgl. auch ebd., VI und dens. 2008, 476: „In diesem Beitrag werden die Daten des Movsēs nicht berücksichtigt."

61 Ich beziehe mich im folgenden auf Werlen 2013a, dessen Aufsatz eine konzentrierte Zusammenfassung seiner einschlägigen Forschungsbeiträge darstellt. Im übrigen ist die Diskussion weiterhin im Fluß. Schon der Faszikel 24.1 (2013) der Zeitschrift „Erwägen Wissen Ethik“, in dem der genannte Text erschienen ist, ist ganz der kritischen Diskussion von Werlens Theorie gewidmet, auf die er mit Werlen 2013b erste Antworten gibt; aus geschichtswissenschaftlicher Sicht einschlägig ist dabei der Beitrag von Rau 2013. Seither hat Werlen sein Konzept einer handlungszentrierten Geographik stets weiterentwickelt und präzisiert; vgl. etwa dens. 2015, 2017 u. 2020. 
de nicht um die „Naturalisierung sozial-kultureller Wirklichkeiten“62 durch raumwissenschaftliche Zugriffe (im Sinne des spatial turn, aber auch älterer Konzepte wie der longue durée bei Fernand Braudel); das „Handeln der Subjekte“, die „subjektive und soziale Welt" ${ }^{\text {663 }}$ steht vielmehr im Vordergrund. Mit Werlens Worten: „Statt alle Bereiche auf den physisch-materiellen zu reduzieren und alle Aspekte erdräumlich darstellen zu wollen, ist zuerst zu klären, welche Rolle (erd-)räumliche Bezüge für unterschiedliche Formen sozialen Handelns spielen." 64

,Raum“ ist laut Werlen kein Apriori, sondern beruht auf Erfahrungen, die die eigentlichen Handlungsmöglichkeiten und -unmöglichkeiten mitbestimmen. Das Subjekt zieht hieraus Konsequenzen durch Etablierung von Bezügen und Bindungen. „Regionalisierung“ (regionalization) ist folglich laut Werlen das Resultat alläglichen Geographie-Machens bzw. die Konsequenz aus der Erschließung der sinnhaften Konstruktion geographischer Wirklichkeiten. ${ }^{65}$ Hervorzuheben ist die Betonung der Interaktion, der Kommunikation durch Werlen. „Handeln gewinnt gegenüber dem Lebensraum [...] Vorrang. "66 Die stete Neuverhandlung der ,gesellschaftlichen Raumverhältnisse“ konstituiert die Gesellschaft mit, ist geeignet, sie zu verändern, indem sie dazu beiträgt, neuen „sozial-kulturellen Wirklichkeiten“ den Weg zu bereiten. ${ }^{67}$

Aus meiner Sicht besteht der Mehrwert von Werlens Theorie in dreifacher Hinsicht: Erstens, sie zeigt nicht nur auf, wie eng ,Raum ' und ,Gesellschaft' aufeinander bezogen sind, sondern zieht aus dieser Erkenntnis auch Konsequenzen. Werlen stellt die Erfahrungen der Menschen mit dem Raum in den Mittelpunkt. Daraus resultiert ihr Handeln. Werlen beschreibt, zweitens, eine Wechselwirkung zwischen ,Mensch' und ,Raum. Durch ihr Handeln gestalten die Menschen den sie umgebenden Raum, sie konstituieren ihn gleichsam in einem fort neu. Werlen spricht von „Formen alltäglichen GeographieMachens“. ${ }^{68}$ Ein Ergebnis dieser menschlichen Tätigkeit ist die „Regionalisierung“ (regionalization). Drittens, dieser Prozeß der Wechselwirkung zwischen ,Mensch “ und ,Raum“ ist nie abgeschlossen. Durch Kommunikation und Interaktion werden die ,gesellschaftlichen Raumverhältnisse" ${ }^{\text {"69 }}$ ständig gestaltet, modifiziert, neukonstituiert. Dabei liegt der Fokus Werlens aber immer auf dem Handeln der Menschen.

Wie läßt sich Werlens Theorie nun für unseren konkreten Fall - Armenien und den südlichen Kaukasus -, fruchtbar machen $?^{70}$ Angesichts der über lange Zeit verhältnismäBig stabilen Verhältnisse - in geographischer, sozialer, sogar makropolitischer Hinsicht ist es nicht verwunderlich, daß alle unsere Quellen, auch die armenischen und georgischen, strukturelle Gemeinsamkeiten aufweisen. Die Interaktion zwischen ,Mensch“ und ,Raum`hat sozusagen immer wieder ähnliche Probleme und Lösungen hervorgebracht.

62 Werlen 2013a, 6.

63 Ebd., 7.

64 Ebd.

65 Ebd., $12 \mathrm{f}$.

66 Ebd., 15.

67 Ebd.

68 Ebd., 11.

69 Ebd., 14.

70 Ganz im Sinne von Rau 2013: „Ein geschichtswissenschaftlicher Aufsatz zum Thema würde vermutlich [...] versuchen, eine Sozialtheorie - in möglichst fruchtbarer Weise - auf eine konkrete historische Fragestellung anzuwenden.“ 
Die historischen Schicksale Armeniens und Iberiens sind eng aufeinander bezogen. Historische Ereignisse, die Armenien betreffen, schlagen früher oder später immer auch auf Iberien durch. Das Gesagte trifft im übrigen in gewisser, allerdings abgeschwächter Weise auch auf Albanien, nicht aber auf Kolchis (Egrisi) und die östliche Schwarzmeerküste zu. Hier ist durch die Gründung griechischer Kolonien und Handelsstützpunkte seit der archaischen Zeit ein nachhaltiger Impuls von außen, aus dem östlichen Mittelmeerraum, erfolgt, der dem meerabgewandten Iberien fehlt.

Die historischen Ereignisse erfolgen im Hinblick auf Iberien immer entlang derselben Kommunikationslinien:

- nach Norden über den $\mathrm{Pa}$ von Darial (weniger über den Paß von Derbent) ins Reiternomadenland nördlich des Kaukasus;

- nach Süden über die breite Kontaktzone entlang der armenisch-iberischen Grenze (bes. Gogarene) nach Armenien und weiter nach Mesopotamien.

- Nach Westen kann die Kommunikationslinie zum römischen Reich direkt aktiviert werden (man denke an den ,Staatsbesuch' Pharasmanes' II. in Rom 141 n. Chr.); meist geschieht dies aber via Armenien (Richtung Kappadokien).

- Nach Osten kann die Kommunikationslinie zum parthischen bzw. persischen Reich ebenfalls direkt aktiviert werden (die iberischen Könige zählen als šahrdārān zu den führenden Dynasten des sāsānidischen Großkönigs); oft geschieht dies aber ebenfalls via Armenien (Richtung Mesopotamien) oder Albanien (Richtung Atropatene). Die Perspektive unserer armenischen und georgischen Quellen reicht potentiell bis nach Zentralasien und Indien.

Die Gültigkeit dieser Kommunikationslinien wird von der griechisch-römischen, der mittelpersischen und parthischen sowie der armenischen und georgischen Überlieferung bestätigt, ungeachtet ereignisgeschichtlicher Anachronismen, offenkundiger ,Fehler ${ }^{6}$ oder schierer ,Erfindungen“. Sie transportieren eine implizite historische Wahrheit, ${ }^{71}$ der gegenüber die Historizität eines armenischen Königs Chosroës II. zweitrangig erscheint.

Dieselbe implizite Wahrheit repräsentieren die inneren und äußeren Verhältnisse, die unsere Quellen, auch die armenischen und georgischen, für Armenien und Iberien schildern. Am Beispiel der sāsānidischen Invasion um 252/62 n. Chr. ist dies hinreichend deutlich geworden: Die Könige erweisen sich letztendlich als Protegés (Klienteloder Vasallenkönige) der Großmächte, seien dies römische Kaiser oder parthische bzw. persische Großkönige. Entsprechend können sie bei militärischen Konflikten in letzter Konsequenz ein- oder abgesetzt werden. Die Könige sind aber auch integriert in die Adelsstrukturen ihrer eigenen Region und der benachbarten Großreiche. Die Auseinandersetzungen von 252/62 n. Chr. haben den Charakter eines erbitterten Adelskonfliktes im Gefolge des Sturzes der Arsakiden 224 n. Chr. Die implizite historische Wahrheit, die unsere armenischen und georgischen Quellen in diesem Zusammenhang korrekt illustrieren, ist die einer permanenten Selbstbehauptung der adeligen Milieus Armeniens

71 Der Begriff ,implizite historische Wahrheit‘ ist in Anlehnung an Assmann 2013, 76 gebildet, der eine ähnliche Formulierung im Hinblick auf die Rolle des Mythos in alten Kulturen gewählt hat: „Mythos ist eine Geschichte, die man sich erzählt, um sich über sich selbst und die Welt zu orientieren, eine Wahrheit höherer Ordnung (Hervorhebung durch mich), die nicht einfach nur stimmt, sondern darüber hinaus auch noch normative Ansprüche stellt und formative Kraft besitzt.“ 
und Iberiens gegenüber den Großmächten, sei es durch ritterlichen Kampf oder ehrenvolle Bündnisse.

Es ist auffallend, daß die Bindung an die iranische Welt bis hin nach Zentralasien und Indien in unseren armenischen und georgischen Quellen grundsätzlich stärker akzentuiert wird als diejenige an die griechisch-römische mediterrane Welt. Mit dem 4. Jh. n. Chr. ändert sich dies in gewisser Weise durch die Christianisierung der Königshäuser von Armenien (um 314 n. Chr.), Iberien und Albanien (einige Jahrzehnte später: unter Constantius II.?). ${ }^{72}$ Die Übernahme des Christentums war keinesfalls selbstverständlich. Man könnte sie mit Werlen, der ja das Handeln der Menschen in den Mittelpunkt seiner Theorie stellt, als eine neue Antwort auf die sozialgeographisch bedingte Prägung des südkaukasischen Raumes interpretieren. Die Privilegierung östlicher, iranischer Einflüsse wurde in der Situation der Christianisierung gleichsam nach Westen umgekehrt. Daraus ergaben sich sowohl positive als auch negative Folgen für die südkaukasischen Reiche.

Positiv ist sicher eine wirksamere Bindung an das spätantike römische Reich, das nun in viel stärkerer Weise seinen Klientelstaaten im südlichen Kaukasus verpflichtet war als früher. Das vierzigjährige hartnäckige Ringen Kaiser Justinians um Lazika im 6. Jh. n. Chr. ist Ausdruck dessen. ${ }^{73}$ Positiv sind auch gewisse kulturelle Begleiterscheinungen: Der Christianisierung folgen die Erfindung der Alphabetschrift und die Schaffung eines einheimischen, darunter auch historischen Schrifttums in altarmenischer, altgeorgischer und kaukasisch-albanischer Sprache. ${ }^{74}$

Aber politisch wogen die Kollateralschäden der Entscheidung, die die Könige Tiridates III., Mirian III. und Urinayr im Verlaufe des 4. Jhs. n. Chr. trafen, doch schwer. Das Imperium Romanum konnte ihre Reiche nicht dauerhaft gegen die Sāsāniden beschützen; sie wurden, im Mahlstrom der konkurrierenden Großmachtinteressen zusehends geschwächt, ab 640 n. Chr. dann leichte Beute der muslimischen Araber.

\section{Zusammenfassung}

Die engen Bezüge zwischen der armenischen und der iberischen Geschichte zwischen dem 1. Jh. v. Chr. und dem 3. Jh. n. Chr. dürften deutlich geworden sein; sowohl die griechisch-römischen als auch die iranischen sowie die armenischen und georgischen Quellen bezeugen sie. Der Großkonflikt zwischen Rom und dem Parther- bzw. Sāsānidenreich ist in der Regel der Rahmen, in den unsere Quellen die Beziehungen zwischen Armenien und Iberien einordnen. Dies bedeutet ohne Zweifel eine Verengung des Blickwinkels, auch für uns Heutige.

72 Zur Christianisierung des Kaukasus und den damit zusammenhängende Forschungsproblemen siehe die Beiträge des Sammelbandes von Seibt 2002; ferner Thomson 1996b und Maraval 2005, 1085 ff. Vgl. auch mit Bezug auf Armenien - Kettenhofen 1995, 74 ff.

73 Hierzu nun Stickler 2019 mit weiterführenden Literaturhinweisen; vgl. auch Braund 1994, 268 ff.

74 Die Zusammenhänge zwischen der Christianisierung und der Schaffung von Alphabeten für die Sprachen des kaukasischen Raumes erschließt an verschiedenen Beispielen der Sammelband von Seibt - Preiser-Kapeller 2011. 
Andererseits treten dadurch die Grundgegebenheiten, unter denen sich die armenische bzw. iberische Geschichte in dem betreffenden Zeitraum vollzog, nur um so schärfer hervor:

- die strukturelle Abhängigkeit der südkaukasischen Reiche von den Großmächten,

- ihre Eingebundenheit in überregionale Konfliktlagen, die sich als regionale Adelskonflikte fortsetzten,

- schließlich die stets wiederkehrenden Muster, denen der ereignisgeschichtliche Verlauf folgte.

Eine unabhängige, gleichsam ,nationale‘ Politik der Könige Armeniens und Iberiens ist vor diesem Hintergrund undenkbar. Auch die armenische und georgische Überlieferung bezeugt dieses Faktum, implizit und bisweilen sogar explizit. Die Christianisierung Armeniens, Iberiens und Albaniens im Verlaufe des 4. Jhs. n. Chr. zeigt die historischen Spielräume, über die die Reiche des südlichen Kaukasus dennoch verfügten. Obwohl sie auf den ersten Blick einen Bruch mit der jahrhundertelang eingeübten (politischen und kulturellen) ,Uneindeutigkeit‘ Armeniens und Iberiens im Verhältnis zu Rom und Iran darzustellen scheint, läßt sie sich doch, nicht zuletzt mit Werlens Theorie einer handlungszentrierten Sozialgeographie, schlüssig in das in diesem Beitrag entwickelte Gesamtbild integrieren.

Diese ,Uneindeutigkeit‘ setzt sich im übrigen, wie hoffentlich deutlich geworden ist, an anderer Stelle fort. Die im Gefolge der Christianisierung geschaffene Schriftkultur in Armenien und Iberien hat eine Form von Geschichtsschreibung hervorgebracht, die in charakteristischer Weise das griechisch-römische Literaturgenus der Historiographie mit Elementen der iranischen Traditionsbildung verbindet. Wenn dieser Zusammenhang gesehen und berücksichtigt wird, kann man vermeiden, an die kaukasische Überlieferung Erwartungen heranzutragen, die sie nicht erfüllen und umgekehrt Fragen an sie richten, zu deren Klärung sie sehr wohl beitragen kann.

\section{LITERATURVERZEICHNIS}

Assmann, J. (2013), Das kulturelle Gedächtnis. Schrift, Erinnerung und politische Identität in frühen Hochkulturen $^{7}$, München.

Bäbler, B. (2019), Pompeius im Kaukasus. Geographie und Topographie eines Feldzugs, in: F. Schleicher u. a. (Hrsgg.), Iberien zwischen Rom und Iran. Beiträge zur Geschichte und Kultur Transkaukasiens in der Antike, Stuttgart: 15-24.

Bosworth, A. B. (1997), Arrian and the Alani, HSCP 81: 217-255.

Braund, D. (1994), Georgia in Antiquity: A History of Colchis and Transcaucasian Iberia 550 BC$A D$ 562, Oxford.

Cereti, C.G. (2001), La letteratura pahlavi. Introduzione ai testi con riferimenti alla storia degli studi e alla tradizione manoscritta, Milano.

Chaumont, M.-L. (1976), L'Arménie entre Rome e l'Iran. De l'avènement d'Auguste à l'avènement de Dioclétien, $A N R W$ 2.9.1: 71-194.

Christensen, A. (1944), L'Iran sous les Sassanides², København. 
Cinemre, İ. T. (2019), The Rise of Armenian Historiography in the Late Antiquity: Mythology and History, Journal of History Culture and Art Research 8.2: 1-12.

Coert, J., Schmitt, T. (2019), Wer war Fl. Dades? Überlegungen zum Verständnis einer Inschrift aus dem kaukasischen Iberien, in: V. Cojocaru u. a. (Hrsgg.), Advances in Ancient Black Sea Studies: Historiography, Archaeology and Religion, Cluj-Napoca: 347-390.

Dąbrowa, E. (1989), Roman Policy in Transcaucasia from Pompey to Domitian, in: D. H. French, Ch. S. Lightfoot (Hrsgg.), The Eastern Frontier of the Roman Empire, Bd. I, Oxford: 67-76.

Daryaee, T. (2001-2002), Memory and History: The Construction of the Past in Late Antique Persia, Nāme-ye Irān-e Bāstan: 1-14.

Garsoïan, N. G. (1982), The Iranian Substratum of the „Agat'angełos“ Cycle, in: N. G. Garsoïan u. a. (Hrsgg.), East of Byzantium: Syria and Armenia in the Formative Period, Washington, D. C.: 151174.

Garsoïan, N. G. (1994), Reality and Myth in Armenian History, in: The East and the Meaning of History, Roma: 117-145 (zit. nach: Garsoïan 1999, XII).

Garsoïan, N. G. (1996), The Two Voices of Armenian Mediaeval Historiography: The Iranian Index, Studia Iranica 25: 7-43 (zit. nach: Garsoïan 1999, XI).

Garsoïan, N. G. (1997), The Aršakuni Dynasty (A. D. 12-[180?]-428), in: R. G. Hovannisian (Hrsg.), The Armenian People from Ancient to Modern Times, Bd. 1: The Dynastic Periods: From Antiquity to the Fourteenth Century, Basingstoke-London: 63-94.

Garsoïan, N. G. (1999), Church and Culture in Early Medieval Armenia, Aldershot.

Giardina, A. (1996), Roma e il Caucaso, in: Il Caucaso. Cerniera fra culture dal Mediterraneo alla Persia (secoli $I V-X I)$, Bd. 1, Spoleto: 85-141.

Goltz, A., Hartmann, U. (2008), Valerianus und Gallienus, in: K.-P. Johne (Hrsg.), Die Zeit der Soldatenkaiser, 2 Bde., Berlin: 223-295.

Hämeen-Anttila J. (2018), Khwadāynāmag, The Middle Persian Book of Kings, Leiden-Boston.

Hartmann, U. (2019), Iberien in der Historia Augusta, in: F. Schleicher u. a. (Hrsgg.), Iberien zwischen Rom und Iran. Beiträge zur Geschichte und Kultur Transkaukasiens in der Antike, Stuttgart: 25-68.

Heftner, H. (1995), Plutarch und der Aufstieg des Pompeius. Ein historischer Kommentar zu Plutarchs Pompeiusvita, Teil I: Kap. 1-45, Frankfurt a. M.

Heid, St. (1991), Zur frühen Protonike- und Kyriakoslegende, Analecta Bollandiana 109: 73-108.

Heil, M. (1997), Die orientalische Außenpolitik des Kaisers Nero, München.

Hoyland, R. G. (2018), The "History of the Kings of the Persians" in Three Arabic Chronicles: The Transmission of the Iranian Past from Late Antiquity to Early Islam, Liverpool.

Huttner, U. (2008), Von Maximinus Thrax bis Aemilianus, in: K.-P. Johne (Hrsg.), Die Zeit der Soldatenkaiser, 2 Bde., Berlin: 161-221.

Huyse, Ph. (2008), Late Sasanian Society between Orality and Literacy, in: V. S. Curtis, S. Stewart (Hrsgg.), The Sasanian Era, London-New York: 140-155.

Johne, K.-P. (Hrsg.) (2008), Die Zeit der Soldatenkaiser, 2 Bde., Berlin.

Kettenhofen, E. (1995), Tirdād und die Inschrift von Paikuli. Kritik der Quellen zur Geschichte Armeniens im späten 3. und frühen 4. Jh. n. Chr., Wiesbaden.

Kettenhofen, E. (2008), Die kaukasischen Reiche, in: K.-P. Johne (Hrsg.), Die Zeit der Soldatenkaiser, 2 Bde., Berlin: 475-500.

Kühnert, F. (1980), Die Orientberichte des Tacitus über Iberien und Armenien, Georgica 3: 36-40.

Maraval, P. (2005), Die neuen Grenzen, in: Ch. Pietri, L. Pietri (Hrsgg.), Die Geschichte des Christentums. Religion, Politik, Kultur. Altertum, Bd. 2: Das Entstehen der einen Christenheit (250-430), Freiburg: 1076-1095.

Markwart, J. (1931), Iberer und Hyrkanier, Caucasica 8: 78-113.

Mosig-Walburg, K. (2009), Römer und Perser vom 3. Jahrhundert bis zum Jahr 363 n. Chr., Frankfurt.

Pourshariati, P. (2008), Decline and Fall of the Sasanian Empire: The Sasanian-Parthian Confederacy and the Arab Conquest of Iran, London-New York. 
Preud'homme, N. (2019), Rois et royauté en Ibérie du Caucase. Entre monde romain et monde iranien. De l'époque hellénistique au début du Ve siècle de notre ère, Diss. Sorbonne Université.

Rapp, St. H. Jr. (2003), Studies in Medieval Georgian Historiography: Early Texts and Eurasian Contexts, Löwen.

Rapp, St. H. Jr. (2014), The Sasanian World through Georgian Eyes: Caucasia and the Iranian Commonwealth in Late Antique Georgian Literature, Farnham.

Rau, S. (2013), Gesellschaft und Raum: Raumverhältnisse historischer Gesellschaften. Ein Kommentar aus geschichtswissenschaftlicher Perspektive, Erwägen Wissen Ethik 24: 54-56.

Schleicher, F. u. a. (Hrsgg.), (2019), Iberien zwischen Rom und Iran. Beiträge zur Geschichte und Kultur Transkaukasiens in der Antike, Stuttgart.

Seibt, W. (Hrsg.) (2002), Die Christianisierung des Kaukasus, Wien.

Seibt, W., Preiser-Kapeller, J. (Hrsgg.) (2011), Die Entstehung der kaukasischen Alphabete als kulturhistorisches Phänomen, Wien.

Shahbazi, A. Sh. (1990), On the X'vadāy-nāmag, in: D. Amin u. a. (Hrsgg.), Iranica varia. Papers in Honor of Professor Ehsan Yarshater, Leiden: 208-229.

Shahbazi, A. Sh. (2004), Historiography II: Pre-Islamic Period, EI 12: 325-330.

Stickler, T. (2019), Der transkaukasische Kriegsschauplatz bei Prokop, in: F. Schleicher u. a., Iberien zwischen Rom und Iran. Beiträge zur Geschichte und Kultur Transkaukasiens in der Antike, Stuttgart: $153-177$.

Terian, A. (2001-2002), Xorenac'i and Eastern Historiography of the Hellenistic Period, Revue des Études Arméniennes 28: 101-141.

Thomson, R. W. (Hrsg.) (1976), Agathangelos: History of the Armenians, Albany.

Thomson, R. W. (Hrsg.) (1978), Moses Khorenats 'i, History of the Armenians, Cambridge, Mass.London.

Thomson, R. W. (1982), The Formation of the Armenian Literary Tradition, in: N. G. Garsoïan u. a. (Hrsgg.), East of Byzantium: Syria and Armenia in the Formative Period, Washington, D. C.: 135150.

Thomson, R. W. (1996a), Rewriting Caucasian History. The Medieval Armenian Adaptation of the Georgian Chronicles. The Original Georgian Texts and the Armenian Adaptation, Oxford.

Thomson, R. W. (1996b), The Origins of Caucasian Civilization. The Christian Component, in: R. G. Suny (Hrsg.), Transcaucasia, Nationalism, and Social Change: Essays in the History of Armenia, Azerbaijan, and Georgia², Ann Arbor: 25-43.

Thomson, R. W. (1996c), The Writing of History: The Development of the Armenian and Georgian Traditions, in: Il Caucaso. Cerniera fra culture dal Mediterraneo alla Persia (secoli IV-XI), Bd. 1, Spoleto: 493-514.

Traina, G. (2002), L'imperatore Probo nella tradizione armena, in: J.-M. Carrié, R. Lizzi (Hrsgg.), Humana sapit. Études d'antiquité tardive offertes à Lellia Cracco Ruggini, Turnhout: 455-467.

Traina, G. (2007), Moïse de Khorène et l'Empire sassanide, in: R. Gyselen (Hrsg.), Des Indo-Grecs aux Sassanides. Données pour l'histoire et la géographie historique, Bures-sur-Yvette: 158-179.

Traina, G. (2015), Tradition et innovation dans la première historiographie arménienne, in: Ph. Blaudeau, P. Van Nuffelen (Hrsgg.), L'historiographie tardo-antique et la transmission des savoirs, Berlin-Boston: 153-164.

Traina, G. (2018), The Rise of the Sasanians: The Armenian Evidence, in: T. Daryaee (Hrsg.), Sasanian Iran in the Context of Late Antiquity, Irvine: 107-119.

Vidman, L. (1982), Fasti Ostienses ${ }^{2}$, Praha.

Werlen, B. (2013a), Gesellschaft und Raum: Gesellschaftliche Raumverhältnisse. Grundlagen und Perspektiven einer sozialwissenschaftlichen Geographie, Erwägen Wissen Ethik 24.1: 3-16.

Werlen, B. (2013b), Weltbilder und Weltsichten. Von disziplinärer Repräsentation zu transdisziplinären Problemstellungen, Erwägen Wissen Ethik 24.1: 76-91. 
Werlen, B. (2015), From Local to Global Sustainability: Transdisciplinary Integrated Research in the Digital Age, in: B. Werlen (Hrsg.), Global Sustainability: Cultural Perspectives and Challenges for Transdisciplinary Integrated Research, Cham: 3-17.

Werlen, B. (2017), Knowledge, Action, and Social Relations of Space, in: P. Meusburger u. a. (Hrsgg.), Knowledge and Action: 31-56.

Werlen, B. (2020), World-Relations and the Production of Geographical Realities: On Space and Action, City and Urbanity, in: J. Rüpke, S. Rau (Hrsgg.), Religion and Urbanity: Reciprocal Formations, Berlin.

Wheeler, E. L. (2017), Roman-Armenian Borders, Part I: The Upper Euphrates Frontier, in: N. Hodgson u. a. (Hrsgg.), Roman Frontier Studies 2009, Oxford: 160-168.

Winter, E., Dignas, B. (2001), Rom und das Perserreich. Zwei Weltmächte zwischen Konfrontation und Koexistenz, Berlin.

Yarshater E. (1983), Iranian National History, in: E. Yarshater (Hrsg.), The Cambridge History of Iran, Bd. 3.1: The Seleucid, Parthian and Sasanian periods, Cambridge: 359-477. 